\title{
ESTIMULAÇÃO MAGNÉTICA TRANSCRANIANA
}

\author{
Adriana B. Conforto', Suely K.N. Marie², Leonardo G. Cohen ${ }^{3}$, Milberto Scaff
}

\begin{abstract}
RESUMO - A estimulação magnética trancraniana (EMT) é técnica não invasiva de investigação e modulação da excitabilidade cortical em humanos. Alterações de excitabilidade cortical em circunstâncias fisiológicas e patológicas podem ser avaliadas através de medidas como limiar motor, potencial evocado motor, curvas de recrutamento, inibição e facilitação intracorticais. O tempo de condução motora central pode estimar a transmissão de impulsos neurais em vias motoras. Mudanças em áreas de representação do córtex sensorimotor podem ser estudadas por mapeamento cortical. A modulação do processamento de diferentes áreas corticais, através da EMT, possibilita o estudo de diferentes funções cerebrais. Aplicações terapêuticas da EMT em depressão, doença de Parkinson e epilepsia têm atraído grande interesse na última década. A integração de EMT a técnicas neurofisiológicas e de neuroimagem oferece possibilidades promissoras de mapeamento cortical não invasivo. A EMT apresenta grande potencial como instrumento de investigação e tratamento em neurologia e em psiquiatria.
\end{abstract}

PALAVRAS-CHAVE: estimulação magnética transcraniana, plasticidade neural, mapeamento cerebral.

\section{Transcranial magnetic stimulation}

ABSTRACT - Transcranial magnetic stimulation (TMS) allows non-invasive study and modulation of cortical excitability in humans. Changes in cortical excitability in physiological and pathological conditions can be tracked by measurements such as motor threshold, motor evoked potentials, recruitment curves, intracortical facilitation and inhibition. The central motor conduction time can estimate neural transmission in central motor pathways. Changes in areas of representation in sensorimotor cortex can be studied with cortical mapping. Modulation of cortical processing can be used to evaluate different brain functions. Therapeutic use in depression, Parkinson's disease and epilepsy has raised great interest over the past decade. Noninvasive cortical mapping may be achieved by combining TMS to other neurophysiological/ neuroimaging techniques. TMS has great potential both as an investigational and as a therapeutical tool in Neurology and Psychiatry.

KEY WORDS: transcranial magnetical stimulation, neural plasticity, brain mapping.

Pesquisas sobre os efeitos de campos magnéticos no sistema nervoso central e periférico datam do século XIX'1, porém somente em 1985 Barker e colaboradores ${ }^{2}$ foram capazes de executar a estimulação magnética transcraniana (EMT) do córtex motor em humanos. Desde então, o emprego da EMT como ferramenta na investigação da fisiologia e fisiopatologia do sistema nervoso tem assumido grande importância. Atualmente, há vários grupos estudan- do aplicações clínicas desta técnica em neurologia e em psiquiatria.

Princípios de estimulação magnética transcraniana

Como é possível realizar a estimulação magnética do córtex cerebral, de forma não invasiva? Inicialmente, coloca-se uma bobina sobre o couro cabeludo (Fig 1). A bobina é conectada a um capacitor através de um circuito elétrico. Quando o circuito é ligado, um pulso de corrente

\footnotetext{
${ }^{1}$ Médica assistente, Divisão de Clínica Neurológica, Hospital das Clínicas/ Faculdade de Medicina da Universidade de São Paulo (HC/ FMUSP), São Paulo SP, Brasil; ${ }^{2}$ Livre-docente, Divisão de Clínica Neurológica, HC/FMUSP; ${ }^{3}$ Chefe da "Human Cortical Physiology Section, Medical Neurology Branch, National Institutes of Neurological Diseases and Stroke, National Institutes of Health", Bethesda, Maryland, USA; ${ }^{4}$ Professor Titular, Diretor da Divisão de Clínica Neurológica, HC/FMUSP.
}

Recebido 16 Maio 2002, recebido na forma final 24 Julho 2002. Aceito 13 Agosto 2002.

Dra. Adriana B. Conforto - Divisão de Clínica Neurológica, HC/FMUSP. Avenida Dr. Enéas de Carvalho Aguiar 255 - 05403000 São Paulo SP - Brasil. E-mail: abconf@yahoo.com 
(cuja intensidade máxima é de cerca de 5000 Amperes) passa rapidamente pela bobina. Em seguida, a corrente é desligada. A mudança rápida na intensidade do campo elétrico promove a indução de um campo magnético, com linhas de fluxo perpendiculares à bobina. A intensidade do campo magnético varia de acordo com os parâmetros do equipamento utilizado, mas em geral é de aproximadamente1,5-2,2 Tesla, nas proximidades da bobina. A intensidade máxima do campo é atingida em cerca de $100 \mu \mathrm{s}$, caindo rapidamente após este intervalo de tempo. A mudança rápida na intensidade do campo magnético promove, dentro do crânio, a indução de um novo campo elétrico, perpendicular ao campo magnético. Em um meio condutor homogêneo, o campo elétrico faria com que fluíssem correntes em um plano paralelo ao da bobina. No córtex, os meios condutores são estruturas neurais localizadas em direção aproximadamente paralela à da bobina, a uma profundidade de 1,5-2 cm abaixo da superfície. O campo induzido no tecido cerebral é de cerca de 100 $\mathrm{mV} / \mathrm{mm}$. Quanto maior a intensidade do campo no córtex cerebral, maior a extensão da despolarização de membranas celulares e, consequentemente, maior a área de ativação neuronal. Como o crânio e o couro cabeludo apresentam alta resistência elétrica, a corrente que flui nestas estruturas é mínima, diminuindo o desconforto que poderia ser causado por excitação de terminações nociceptivas ${ }^{1-9}$.

Há várias técnicas de EMT, com diferentes objetivos, como a investigação de mecanismos fisiológicos e fisiopatológicos corticais - particularmente relacionados à motricidade, plasticidade e aprendizado - e o tratamento de doenças neurológicas e psiquiátricas ${ }^{1-9}$. A seguir, discutire- mos os principais métodos de EMT - estimulação com pulso único, com pares de pulsos, estimulação repetitiva - e o tipo de informação e/ou efeito obtido com cada um.

\section{Estimulação com pulso único (EMT-p)}

A modalidade de EMT mais amplamente utilizada é a estimulação com pulso único (EMT-p): um pulso de corrente elétrica passa pela bobina, sendo repetido após intervalos variáveis de alguns segundos. O número total de pulsos administrados é definido pelo examinador. Desta forma, são geradas correntes elétricas no córtex cerebral, desencadeando determinados efeitos, dependendo da região estimulada. Por exemplo, a bobina pode ser colocada no couro cabeludo, na topografia do giro pré-central, para estimulação de neurônios do córtex sensorimotor (Fig 1). Desta forma, de acordo com a principal teoria sobre o mecanismo de ação da EMT, são ativados interneurônios situados a pequena distância da superfície cortical e que mantêm conexões sinápticas com o trato córtico-espinhal $(\mathrm{TCE})^{7}$. Dependendo da angulação da bobina sobre o couro cabeludo, é possível que o TCE seja estimulado diretamente ${ }^{3}$. A estimulação indireta ("trans-sináptica") ou direta do TCE, pode promover excitação de circuitos motores medulares e finalmente, de unidades motoras. Através de eletrodos de superfície colocados sobre músculos contralaterais ao hemisfério estimulado, registra-se o potencial evocado motor (PEM) ${ }^{1,5,6}$ (Fig 1) que representa a ativação de fibras musculares das unidades motoras estimuladas. A amplitude do PEM depende, entre outros fatores, da intensidade do estímulo magnético e do grau de contração muscular pré-estimulação ${ }^{3-5}$. Quanto maior a

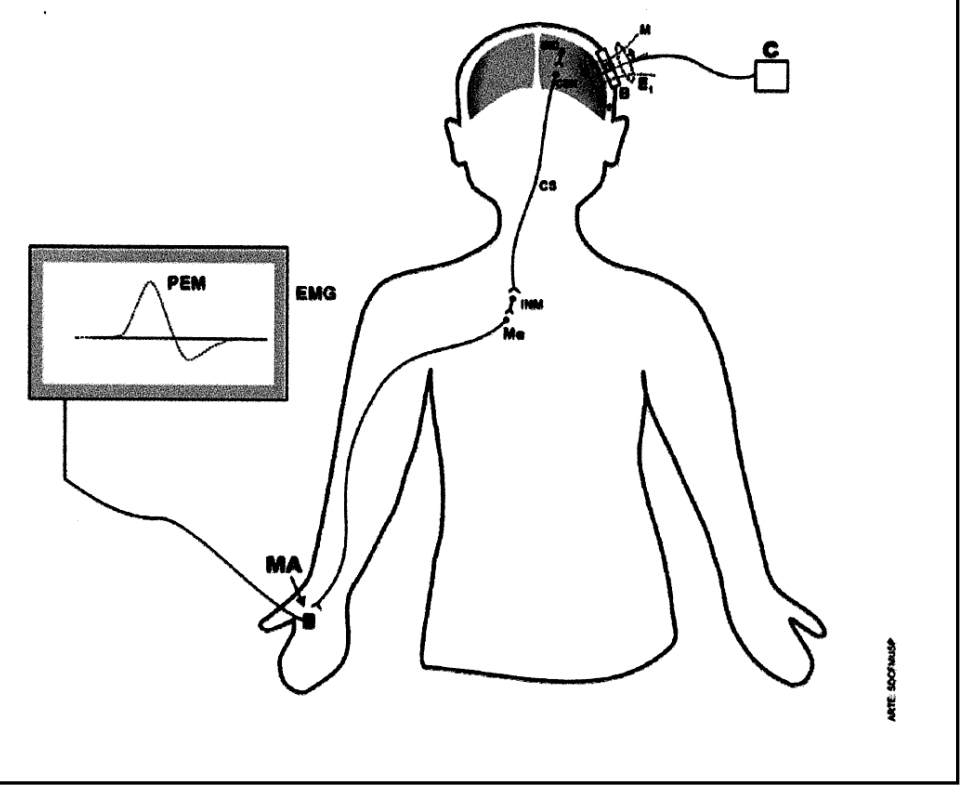

Fig 1. Desenho esquemático do mecanismo de ação da estimulação magnética transcraniana (EMT). C, capacitor; $B$, bobina; $M$, linhas do campo magnético; $E_{1}$, campo elétrico na bobina; $E_{2}$, campo elétrico no córtex; INC, interneurônio no córtex sensorimotor; CSN, corpo celular de neurônio cujo axônio fará parte do trato córtico-espinhal; CS, trato córtico-espinhal; INM, interneurônio medular; $M \alpha$, motoneurônio alfa medular; $M A$, músculo-alvo; EMG, eletromiógrafo; PEM, potencial evocado motor registrado. 
intensidade do campo magnético, maior a amplitude do PEM. Da mesma forma, a contração voluntária do músculo-alvo imediatamente antes da estimulação está associada a aumento na amplitude do PEM - este fenômeno é denominado facilitação ${ }^{3}$. A amplitude pode ser representada por seu valor absoluto em milivolts $(\mathrm{mV})$. Mais frequentemente, a amplitude da resposta $\mathrm{M}$ é registrada por eletromiografia de superfície e a amplitude do PEM é expressa em valor relativo: razão entre amplitude do PEM em $\mathrm{mV}$ e amplitude da resposta $\mathrm{M}$ em $\mathrm{mV}$ (PEM/M, valor percentual).

Dependendo do tipo de bobina, de seu posicionamento no couro cabeludo e da intensidade do campo, áreas restritas do córtex sensorimotor podem ser estimuladas. 0 ponto no couro cabeludo onde a estimulação magnética evoca resposta de amplitude máxima e com maior facilidade em determinado músculo-alvo é considerado o local preferencial, ou "hot spot", para registro do PEM para este músculo. Quando PEMs são obtidos por estimulação de diferentes pontos no crânio, representações corticais de músculos podem ser mapeadas ${ }^{5,10,11}$. A resolução espacial do mapa dependerá, entre outros fatores, do formato da bobina utilizada. A bobina "em forma de 8" é a mais amplamente utilizada para estimulação focal do córtex, com resolução espacial de cerca de $0,5 \mathrm{~cm}^{7}$. Ultimamente, vêm sendo desenvolvidos sistemas estereotáxicos e de neuronavegação para EMT ${ }^{1}$. Regiões específicas no córtex poderiam ser selecionadas precisa e diretamente de cortes de ressonância magnética e suas representações motoras, investigadas.

Outra informação fornecida pela EMT-p é o tempo de condução motora central (TCMC), que corresponde ao tempo de condução do impulso nervoso, do córtex a motoneurônios espinhais. Pode ser estimado pela subtração: (latência total de PEMs) - (tempo de condução em nervos e junção neuromuscular após excitação de raízes motoras no forame intervertebral). Nesta medida estão embutidos o verdadeiro tempo de condução em vias motoras centrais, o tempo de transmissão sináptica a nível espinhal e o tempo da condução da parte proximal da raiz motora ao forame intervertebral, quando estímulos elétricos ou magnéticos são utilizados para a estimulação radicular ${ }^{12}$. Aumentos na duração do TCMC após AVC correlacionamse com sinais piramidais, enquanto normalidade ou aumentos muito discretos do TCMC correlacionam-se com melhor prognóstico de recuperação funcional ${ }^{12}$. A medida do TCMC tem sido também utilizada, em especial na Europa, para diagnóstico e seguimento de pacientes com esclerose múltipla e esclerose lateral amiotrófica (ELA). Ocorre um aumento na duração do TCMC, relacionado à desmielinização dos tratos córtico-espinhais na primeira e à sua degeneração na segunda ${ }^{3,12}$. Além do mapeamento cortical e da medida do TCMC, a estimulação com pulso único fornece informações sobre parâmetros de excitabilidade do córtex motor ${ }^{5-7,9}$ : limiar motor, curvas de recrutamento e período silente, entre outros.

O limiar motor em repouso (LM) corresponde à menor intensidade de estímulo capaz de evocar PEMs de amplitude de no mínimo $50 \mu \mathrm{V}$, após pelo menos 5 dentre 10 pulsos magnéticos administrados, quando o músculo-alvo está em repouso. Acredita-se que o LM represente uma medida da excitabilidade de membrana dos neurônios do trato córtico-espinhal ${ }^{7,9}$. Quanto maior o LM, menor a excitabilidade

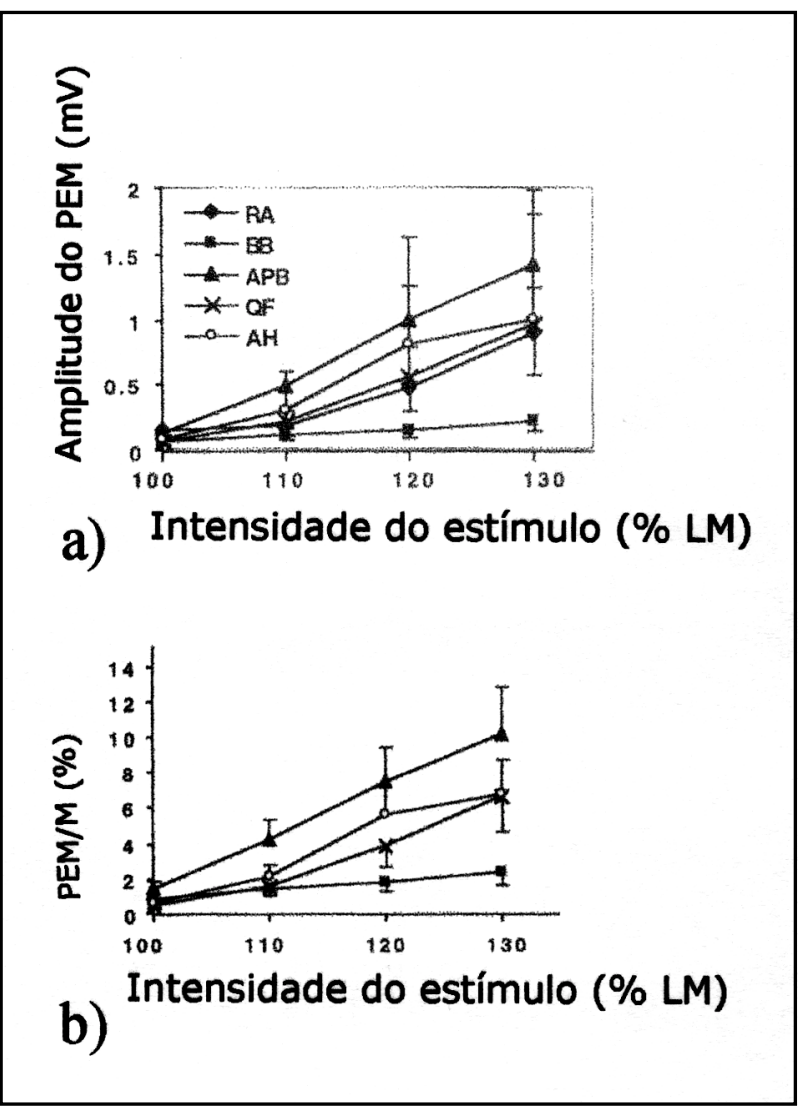

Fig 2. Curvas de recrutamento. $R A$, músculo reto abdominal; $B B$, músculo bíceps braquial; $A P B$, músculo abdutor curto do polegar; $Q F$, músculo quadríceps; $A H$, músculo abdutor do hálux; $L M$, limiar motor em repouso; PEM, potencial evocado motor; $P E M / M$, relação entre a amplitude do potencial evocado motor $e$ a resposta $M$ obtida com eletromiografia de superfície. A intensidade do estímulo magnético está representada pela relação entre a intensidade absoluta do estímulo e a intensidade de estímulo em que se obtém o limiar motor em repouso. À medida em que a intensidade do estímulo é elevada, ocorre aumento da amplitude do PEM, que pode ser representada como amplitude absoluta em milivolts (Fig 2a) ou como relativa à amplitude da resposta $M$ registrada por eletromiografia de superfície (Fig $2 b$ ). Adaptado de Chen R, Tam A, Butefisch C, et al. J Neurophysiol 1998;80:2870-2881. 
neuronal. O LM está aumentado, por exemplo, em doentes portadores de ELA em fase avançada e na fase aguda de acidente vascular cerebral (AVC) ${ }^{3}$. Drogas que bloqueiam canais de sódio, como a carbamazepina e a fenitoína, promovem aumento do LM, enquanto agonistas gabaérgicos, como benzodiazepínicos, não o modificam ${ }^{3,5,7,13}$.

Imediatamente após o registro de PEMs durante contração voluntária do músculo-alvo (PEMcv - potencial evocado motor registrado durante contração voluntária), observa-se habitualmente ausência de atividade eletromiográfica por um intervalo de tempo que é denominado período silente (PS). Quanto maior a intensidade do estímulo magnético, maior a duração do PS. Embora existam controvérsias quanto aos mecanismos fisiológicos do PS,

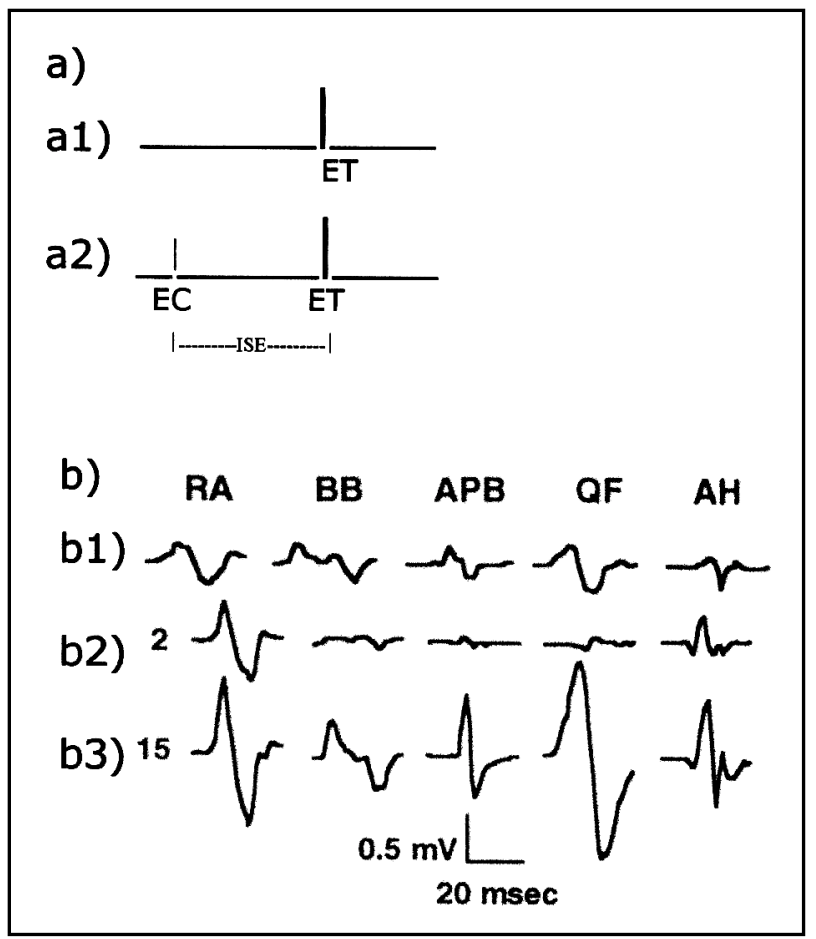

Fig 3. Inibição e Facilitação Intracorticais. 3a) Desenho esquemático da administração de pulsos magnéticos. Em a1, apenas um único pulso (estímulo-tese , ET) é administrado para o registro do PEM. Em a2, o ET é precedido por um estímulo controle (EC), de menor intensidade. ISE, Intervalo entre os 2 pulsos. 3b) Amplitudes de PEM ( $m V$ ) em diferentes músculos após estimulação com pulso único (3b1) e após estimulação com pares de pulsos (3b2 e 3b3). $R A$, músculo reto abdominal; $B B$, músculo bíceps braquial; $A P B$, músculo abdutor curto do polegar; $Q F$, músculo quadríceps; $A H$, músculo abdutor do hálux. Em b2, o ISE é de 2 ms e ocorre diminuição da amplitude do PEM comparada à obtida em b1: Inibição Intracortical. Em b3, o ISE é de 15 ms e ocorre aumento da amplitude do PEM comparada à obtida em a b1: facilitação intracortical. Adaptado de: Chen R, Tam A, Butefisch C, et al. J. Neurophysiol. 1998;80:2870-2881. acredita-se que se relacionem a fenômenos de inibição cortical $^{3,9}$. Anormalidades foram descritas na evolução de ELA, ocorrendo encurtamento progressivo e, às vezes, até mesmo desaparecimento do PS no decorrer da doença ${ }^{3}$. $\mathrm{Na}$ cãimbra do escrivão, verifica-se encurtamento progressivo do PS, à medida em que o doente executa uma contração voluntária cada vez maior do músculo-alvo, o que habitualmente não ocorre em pessoas saudáveis. É possível que esta característica neurofisiológica esteja relacionada a distúrbios em mecanismos inibitórios corticais nesta modalidade de distonia focal $^{3}$. Em alguns pacientes hemiparéticos, após AVC, a duração do PS pode tornar-se proporcional ao grau de contração do músculo-alvo, como em portadores de cãimbra do escrivão. Nestes casos, o prognóstico motor é pior, havendo maior tendência à evolução para espasticidade. Além disso, após AVC, habitualmente ocorre um aumento na duração do $\mathrm{PS}^{3}$.

Pulsos únicos administrados a intensidades progressivamente maiores, aplicados ao córtex motor, podem ser usados para gerar uma curva de recrutamento (CR) (Fig 2). Esta curva reflete o aumento na amplitude do PEM, à medida em que a intensidade da EMT é elevada. Os mecanismos subjacentes às CRs ainda não estão completamente elucidados, mas acredita-se que envolvam a ativação progressiva de neurônios menos excitáveis ou mais distantes do centro de ativação da EMT. As CRs provavelmente traduzem a força de projeção córtico-espinhal para neurônios medulares, ou seja, a proporção de neurônios motores alfa que é recrutada por estimulação magnética cortical de intensidade crescente ${ }^{5,9,14}$.

\section{Estimulação com pares de pulsos (EMT-pp)}

Nesta técnica, dois pulsos magnéticos são administrados consecutivamente, havendo um intervalo de poucos milissegundos (ms) entre eles (Fig 3a). Na modalidade mais amplamente utilizada de EMT-pp, a intensidade do primeiro pulso situa-se abaixo do LM e a do segundo, acima do mesmo. A intervalos menores que cerca de $5 \mathrm{~ms}$ entre os dois pulsos - podendo ser maiores ou menores que 5 ms, dependendo do músculo e das intensidades dos estímulos - habitualmente verifica-se uma diminuição na amplitude do PEM (Fig 3b2), comparada à registrada quando um único pulso é administrado (Fig 3b1). Este fenômeno é denominado inibição intra-cortical (IIC). A intervalos maiores que $5 \mathrm{~ms}$ entre os dois pulsos, ocorre o fenômeno oposto, com aumento da amplitude do PEM (Fig 3b3): é a facilitação intra-cortical (FIC). Acredita-se que estes fenômenos relacionem-se à atividade de diferentes circuitos inibitórios e excitatórios no córtex motor ${ }^{14-16}$.

\section{Aplicações de EMT-p e EMT-pp}

A avaliação de efeitos de drogas anti-epilépticas sobre 
a excitabilidade cortical - com utilização de medidas como o limiar motor e de curvas de recrutamento, através de EMT-p - é uma aplicação promissora da EMT. É possível que mecanismos de epileptogênese relacionados a alterações na excitabilidade neuronal possam ser investigados de forma não invasiva.

EMT-p e EMT-pp têm sido utilizadas para avaliação de excitabilidade neuronal, efeitos de drogas ${ }^{7,13,17}$, evolução e prognóstico em $\mathrm{AVC}^{3,12}$, ELA e esclerose múltipla ${ }^{3}$, além de mapeamento de representações motoras no córtex cerebral. A avaliação do PEM e do TCMC poderia ser útil, também, para diferenciar paresias/ plegias associadas a síndromes psiquiátricas, de lesões de vias motoras centrais.

Ambas as técnicas têm sido amplamente utilizadas na investigação de plasticidade neural ${ }^{18}$. Estudos sobre a reorganização do córtex motor após AVC e em modelos de deaferentação do córtex sensorimotor, por exemplo, têm fornecido importantes informações sobre a plasticidade do córtex adulto em humanos ${ }^{18-21}$.

\section{Estimulação repetitiva (EMT-r)}

A estimulação repetitiva (EMT-r) refere-se à aplicação de estímulos magnéticos a intervalos regulares. Quando são empregadas frequências maiores que $1 \mathrm{~Hz}$, a EMT-r é denominada de alta frequência, ou "rápida" e quando frequências menores são utilizadas, EMT-r de baixa frequência, ou "lenta". Estimuladores especiais, compostos de capacitores múltiplos permitem o uso de frequências de pulsos de até $60 \mathrm{~Hz}^{7}$.

A EMT-r pode modificar o processamento cerebral, sensorimotor ou cognitivo $4,5,7,8,22$, podendo promover disfunções temporárias em diferentes áreas corticais - estimulação temporal e frontal inferior podem levar a afasia transitória, por exemplo ${ }^{8,23}$. O efeito da EMT-r sobre o desempenho de diferentes tarefas tornou esta técnica uma importante ferramenta na investigação do papel fisiológico de diferentes áreas cerebrais. Em geral, EMT-r de baixa frequência promove diminuição temporária de excitabilidade do córtex motor, enquanto o efeito oposto é obtido com EMT-r de alta frequência ${ }^{24}$. Atualmente, estuda-se a aplicabilidade clínica da EMT-r, através de sua interferência na excitabilidade cortical, como discutiremos a seguir.

\section{Aplicações clínicas: EMT-r}

Epilepsia - A possibilidade de substituição do teste de Wada por EMT-r tem sido aventada. O teste de Wada é comumente empregado para determinação da dominância hemisférica para linguagem, no planejamento pré-operatório de epilepsia temporal. Uma vez que a EMT-r pode alterar transitoriamente o funcionamento de áreas de linguagem no hemisfério direito e esquerdo, esta técnica poderia ser utilizada para avaliação de dominância hemisférica ${ }^{7,8,23}$. Até o momento, o teste de Wada permane- ce como padrão-ouro, não sendo substituível pela EMT-r. É possível que a utilização conjunta de EMT-r e Ressonância Magnética Funcional (RMf) permita a determinação não invasiva de dominância para linguagem, no futuro. Alguns autores investigaram o emprego de EMT-r para o tratamento de epilepsia de difícil controle ${ }^{7,25,26}$ e descreveram melhora no controle de crises após EMT-r de baixa frequência. Contudo, estudos ulteriores não confirmaram estes achados e a aplicabilidade terapêutica da EMT-r em epilepsia ainda não está estabelecida.

Movimentos anormais - Pascual-Leone, em 1994, descreveu um aumento na velocidade de reação e no desempenho motor em portadores de doença de Parkinson após EMT-r do córtex motor ${ }^{27}$. Porém, outros estudos não confirmaram estes achados ${ }^{28}$ e a questão permanece em aberto.

Depressão - Nos últimos anos, o foco de maior interesse sobre o uso clínico de EMT tem sido o tratamento da depressão: seria possível substituir a eletroconvulsoterapia (ECT) pela EMT-r? Parte-se do pressuposto de que na depressão ocorra uma hipoatividade do córtex pré-frontal (CPF) esquerdo. A EMT-r de alta frequência do CPF esquerdo, ou EMT-r de baixa frequência do CPF direito poderiam balancear o funcionamento das duas áreas e assim, promover melhora clínica nesta doença ${ }^{8,29}$. Até o momento não há evidências, por ensaios clínicos controlados, de que a EMT-r possa substituir ou mesmo alcançar a eficácia da ECT. Contudo, esta linha de pesquisa vem crescendo e há vários grupos investigando o tema. É possível que, dentro de alguns anos, a EMT-r possa vir a fazer parte do arsenal terapêutico em Psiquiatria.

\section{EMT integrada a outras modalidades de investigação}

Uma das limitações ao emprego diagnóstico e terapêutico da EMT é sua resolução espacial, ou seja, o grau de precisão anatômica alcançado com esta técnica. As principais vantagens são sua resolução temporal e a possibilidade de interferir diretamente em processos fisiológicos no sistema nervoso central, de forma não invasiva. A integração da EMT a outras técnicas como RMf, Magnetencefalografia (MEG), tomografia por emissão de pósitrons (PET), tomografia computadorizada por emissão de fóton único (SPECT) e Eletrencefalografia (EEG) promove o casamento entre informações anatômicas e funcionais, possibilitando melhor compreensão da fisiologia e fisiopatologia corticais. Na prática clínica, a localização precisa de áreas encefálicas seria de grande utilidade, por exemplo, em Neurocirurgia. O conhecimento detalhado da função de determinadas áreas corticais possibilitaria o planejamento da extensão de ressecção de tumores e de outras lesões, minimizando o dano neurológico. A integração entre diferentes técnicas neurofisiológicas e de neuroimagem 
para mapeamento cerebral é hoje uma vertente de pesquisa de ponta em Neurologia e em Psiquiatria ${ }^{6,30,31}$.

\section{Segurança}

EMT-p - Foram definidas diretrizes para o emprego de EMT. A EMT-p é considerada uma técnica segura ${ }^{32}$, desde que as contra-indicações absolutas para seu uso sejam respeitadas: marcapassos cardíacos, aparelhos eletrônicos ou objetos metálicos intracranianos, falhas ósseas no crânio. Relatos de crises convulsivas após EMT-p são raros na literatura, quer em voluntários saudáveis, quer em portadores de doenças neurológicas, com ou sem epilepsia. Mesmo nos casos relatados, não ficou claro se a EMT-p realmente desencadeou as crises epilépticas ${ }^{7}$. Paradoxalmente, vários autores não conseguiram "ativar" focos epilépticos em doentes com crises parciais complexas de difícil controle. Inclusive, foi descrita diminuição da frequência de espículas após $\mathrm{EMT}^{7}$. Nos últimos anos, milhares de pacientes e voluntários foram submetidos a EMT-p em todo o mundo, sem que houvesse desencadeamento de crises epilépticas. Desta forma, considera-se a EMT-p uma técnica bastante segura.

EMT-pp - Não há estudos sistemáticos sobre a segurança de EMT-pp em epilépticos. Autores que têm grande experiência no assunto consideram que a segurança seja equivalente à da EMT-p ${ }^{7}$.

EMT-r - Mesmo pessoas não epilépticas estão sob risco de apresentar crises epilépticas após EMT-r6,33. Em 1996, foram definidas normas de segurança para EMT-r, em uma reunião internacional ${ }^{32}$. Wassermann publicou tabelas de valores de intensidades de estimulação máximas permitidas (tendo como referência o LM) para diferentes frequências de EMT-r , assim como intervalos necessários entre trens de pulsos de EMT-r. No mesmo artigo, encontrase uma revisão bastante extensa sobre possíveis efeitos adversos de EMT, tais como alterações hormonais, imunológicas, comportamentais e auditivas, entre outras. As contra-indicações para emprego da EMT-r são as mesmas descritas para a EMT-p, acrescidas de: hipertensão intracraniana, cardiopatia grave (pelo risco de complicações graves após crises convulsivas); uso de tricíclicos, neurolépticos e outras drogas que diminuem o limiar para crises epilépticas, a não ser que o benefício potencial seja maior que o risco de crise; gestação. Em geral, evita-se a EMT-r em crianças, a não ser na investigação de benefícios clínicos, como no tratamento de epilepsia refratária ou depressão ${ }^{32}$.

\section{CONCLUSÃO}

A EMT-p vem sendo utilizada, para fins de pesquisa, em todo o mundo, e com finalidades clínicas na Europa basicamente, para prognóstico em AVC, diagnóstico e se- guimento em ELA e esclerose múltipla. Nos EUA, seu emprego para tratamento de depressão é realizado através de regulamentação específica para "Investigational Device Exemptions" (IDEs), do FDA. É preciso reconhecer, porém, que a EMT é relativamente recente e que o número de estudos a seu respeito vem crescendo a grande velocidade. A integração da EMT a outras modalidades de investigação em Neurofisiologia e Neuroimagem é, sem dúvida, uma ferramenta promissora de investigação e tratamento em neurologia e psiquiatria.

\section{REFERÊNCIAS}

1. Ilmonemi R, Ruohonen J, Karhu J. Transcranial magnetic stimulation: a new tool for functional imaging of the brain. Critl Rev Biomed Eng 1999;27:241-284.

2. Barker AT, Jalinous R, Freeston IL. Non-invasive magnetic stimulation of the human motor cortex. Lancet 1985;2:1107.

3. Mills KR. Magnetic stimulation of the human nervous system. New York, Oxford University Press, 1999:27-115;155-238.

4. Pascual-Leone A, Tormos JM, Keenan J, Tarazona F, Canete C, Catalá MD. Study and modulation of human cortical excitability with transcranial magnetic stimulation. J Clin Neurophysiol. 1998;15:333-343.

5. Cohen LG, Ziemann U, Chen R, et al. Studies of neuroplasticity with transcranial magnetic stimulation. J Clin Neurophysiol 1998;15:305-324.

6. Hallett M. Transcranial magnetic stimulation of the human brain Nature 2000;406:147-150.

7. Ziemann U, Steinhoff BJ, Tergau F, Paulus W. Transcranial magnetic stimulation: its current role in epilepsy research. Epilepsy Res 1998;30:11-30.

8. Fregni F, Pascual-Leone A. Estimulação magnética transcraniana: uma nova ferramenta para o tratamento da depressão? Rev Psiq Clin. 2001; 28:253-265.

9. Pascual-Leone A, Tormos JM, Keenan J, Tarazona F, Canete C, Catalá MD. Study and modulation of human cortical excitability with transcranial magnetic stimulation. J Clin Neurophysiol. 1998;15:333-343.

10. Wassermann EM, McShane LM, Hallett M, Cohen LG. Noinvasive mapping of muscle representations in human motor cortex. Electroencephalogr Clin Neurophysiol 1992;85:1-8.

11. Brasil-Neto JP, Cohen LG, Panizza M, Nilsson J, Roth BJ, Hallett M. Optimal focal transcranial magnetic ativation of the human motor cortex: effects of coil orientation, shape of the induced current pulse, and stimulus intensity. J Clin Neurophysiol. 1992;9:132-136.

12. Rossini PM, Pauri F. Central motor conduction studies. Electroencephalogr Clin Neurophysiol 1999;51( Suppl):199-211.

13. Ziemann U, Lonnecker S, Steinhoff BJ, Paulus W. Effects of antiepileptic drugs on motor cortex excitability in humans: a transcranial magnetic stimulation study. Ann Neurol. 1996;40:367-378.

14. Chen R. Studies of human motor physiology with transcranial magnetic stimulation. Muscle Nerve 2000;9 (Suppl): S26-S32.

15. Chen R, Tam A, Butefisch C, et al. Intracortical inhibition and facilitation in different representations of the human motor cortex. J Neurophysiol. 1998;80:2870-2881.

16. Ziemann U. Intracortical inhibition and facilitation in the conventional paired TMS paradigm. Electroencephalogr Clin Neurophysiol 1999;51(Suppl):127-136.

17. Stefan K, Kunesch E, Benecke R, Classen J. Effects of riluzole on cortical excitability in patients with amyotrophic lateral sclerosis. Ann Neurol. 2001;49:537-540.

18. Cohen LG, Ziemann U, Chen R. Mechanisms, functional relevance and modulation of plasticity in the human central nervous system. Electroencephalogr Clin Neurophysiol Suppl. 1999;51(Suppl):174-182.

19. Cramer SC, Bastings EP. Mapping clinically relevant plasticity after stroke. Neuropharmacology 2000; 39: 842-851.

20. Ziemann U, Hallett M, Cohen LG. Mechanisms of deafferentationinduced plasticity in human motor cortex. J Neurosci 1998;18:7000-7007.

21. Traversa R, Ciccinelli P, Oliveri M, et al. Neurophysiological followup of motor cortical output in stroke patients. Clin Neurophysiol 2000; 111:1695-1703. 
22. Pascual-Leone A, Hallett M. Induction of errors in a delayed response task by repetitive transcranial magnetic stimulation of the dorsolateral prefrontal cortex. Neuroreport 1994;5:2517-2520.

23. Epstein CM, Lah JJ, Meador K, Weissman JD, Gaitan LE, Dihenia B. Optimum stimulus parameters for lateralized suppression of speech with magnetic brain stimulation. Neurology 1996;47:1590-1593.

24. Chen R, Seitz RJ. Changing cortical excitability with low-frequency transcranial magnetic stimulation. Neurology 2001;57:379-380.

25. Tergau F, Naumann U, Paulus W, Steinhoff B. Low-frequency repetitive transcranial magnetic stimulation improves intractable epilepsy. Lancet 1999;353:2209.

26. Menkes DL, Gruenthal MS. Slow-frequency repetitive transcranial magnetic stimulation ina patient with focal cortical dysplasia. Epilepsia 2000;41:240-242

27. Pascual-Leone A, Valls-Sole J, Brasil-Neto JP, Cammarota A, Grafman J, Hallett M. Akinesia in Parkinson's disease. I. Shortening of simple reaction time with focal, single-pulse transcranial magnetic stimulation. Neurology 1994;44:884-891.
28. Ghabra MB, Hallett M, Wassermann E. Simultaneous repetitive transcranial magnetic stimulation does not speed fine movement in PD. Neurology 1999;52:768-770.

29. Wassermann EM, Lisanby SH. Therapeutic application of repetitive transcranial magnetic stimulation: a review. Clin Neurophysiol. 2001;112:1367-1377.

30. Rossini PM, Pauri F. Neuromagnetic integrated methods tracking human brain mechanisms of sensorimotor areas "plastic" reorganisation. Brain Res Rev 2000;33:131-154.

31. Wassermann EM, Wang B, Zeffiro TA, et al. Locating the motor cortex on the MRI with transcranial magnetic stimualtion and PET. Neuroimage 1996;3:1-9.

32. Wassermann EM. Risk and safety of repetitive transcranial magnetic stimulation: report and suggested guidelines from the International Workshop on the safety of repetitive transcranial magnetic stimulation, June 5-7, 1996. Electroencephalogr Clin Neurophysiol 1998;108:1-16.

33. Pascual-Leone A, Valls-Solé J, Brasil-Neto JP, Cohen LG, Hallett M. Seizure induction and transcranial magnetic stimulation. Lancet 1992;339:997. 